\title{
Serial processing and the parallel-lines illusion: Length contrast through relative spatial separation of contours
}

\author{
KEVIN JORDAN \\ San Jose State University, San Jose, California \\ and \\ DIANE J. SCHIANO \\ Oberlin College, Oberlin, Ohio
}

\begin{abstract}
The distortion of perceived line length produced by the parallel-lines configuration reversed from assimilation to contrast with large suprafoveal spatial separation of the contextual and test lines (Experiment 1). This new contrast effect was predicted by a pool-and-store model of size distortion (Girgus \& Coren, 1982). However, contrary to the predictions of the model, the amount of spatial separation needed to produce the reversal did not depend on an absolute visual-angle (foveal) measure of separation. Rather, the reversal was determined by the relative spatial separation of contextual and test lines (Experiment 2); that is, small test lines required a smaller spatial separation from a contextual line to produce contrast than did larger test lines. On the basis of these findings, a revised pool-and-store model of length distortion is proposed.
\end{abstract}

Perhaps the simplest of all visual size distortions are those that compose the parallel-lines illusion. Indeed, Pressey (1983) has argued that the illusion may serve as a prototype for a wide variety of more popular size distortions, such as the Müller-Lyer, Baldwin, and Ponzo illusions. As shown in Figure 1, the illusion configuration consists of two vertically displaced horizontal lines, typically with the test line presented below the contextual line. A standard test line's length is underestimated when the line is presented with a shorter contextual line and overestimated when it is presented with a longer contextual line. Such distortions of perceived test-line length toward the context are labeled assimilation effects, whereas distortions of test-line length away from the context are labeled contrast effects.

Most size distortions are classified as either assimilation or contrast effects and, as a result, most models of size distortion have focused on describing only assimilation or contrast. For example, Pressey's assimilation theory (e.g., Pressey, 1972) has provided a good description of assimilation in the parallel-lines figure (Pressey \& Murray, 1976), the Müller-Lyer figure (Pressey \& Bross, 1973), and the Ponzo figure (Pressey, Butchard, \& Scrivner, 1971). However, it has recently become clear that many illusion figures produce both assimilation and contrast effects under the appropriate conditions. Pressey and Wilson (1980) reported both assimilation and con-

Preparation of this manuscript was supported by NASA Grant NCC2-327 to San Jose State University, Kevin Jordan, Project Director. Requests for reprints should be sent to Kevin Jordan, Department of Psychology, San Jose State University, San Jose, CA 95192.
Figure 1. Example of the parallel-lines illusion configuration. Relative to a no-context control line, the test line is overestimated when presented with the longer contextual line.

trast in the Baldwin figure and asserted the need for a unified model of assimilation and contrast. Similarly, Brigell and Uhlarik (1979) reported both assimilation and contrast in the parallel-lines figure.

Coren and Girgus (1978; see also Girgus \& Coren, 1982) have attempted to unify explanations of assimilation and contrast of perceived size. According to their pool-and-store model, the critical factor determining the direction of distortion is whether both contextual and test contours are sampled within a single glance. The simultaneous viewing of the contours is assumed to promote perceptual pooling, resulting in assimilation of perceived size. Alternatively, Coren and Girgus propose that when successive fixations are required to view the contextual and test contours, contrast results from differential encoding into memory store. Additionally, whether contextual and test contours are sampled in a single glance or in successive glances depends on the temporal and spatial proximity of the contours. Stimuli presented sequentially (as in the aftereffect paradigm) are sampled in separate glances by definition; stimuli presented simultaneously but with a large intercontour separation are also sampled in separate glances. Thus, both temporal and spa- 
tial separation can produce contrast, according to the model. Finally, Girgus and Coren (1982) state that the criterion for distinguishing large and small intercontour separations is foveal. Subfoveal intercontour separations produce assimilation and suprafoveal separations produce a reversal to contrast.

Brigell and Uhlarik's (1979) results with the parallellines array provide some support for the pool-and-store model. When they presented the contextual and test lines simultaneously and in close spatial proximity, assimilation was observed. Also, when the contextual line was inspected for $60 \mathrm{sec}$ prior to the presentation of a test line in an aftereffect paradigm, contrast resulted. As predicted by the model, assimilation reversed to contrast under conditions requiring sequential sampling of the array. Jordan and Uhlarik (1985) reported that $5 \mathrm{sec}$ of contextual exposure prior to judgment of the test line also produced length contrast in the parallel-lines array. This result is important because it indicates that temporal separation per se, rather than prolonged exposure to the contextual line, is responsible for the shift from assimilation to contrast.

As noted above, the pool-and-store model proposes a functional equivalence of temporal and spatial separation of contextual and test stimuli in producing a shift from length assimilation to contrast, since both kinds of separation require sequential sampling of the array. The present experiments represent a test of the pool-and-store model. If temporal and spatial separation are functionally equivalent, as the model suggests, then length contrast should result from large spatial separations. Experiment 1 established length contrast with large vertical spatial separations of horizontal contextual and test lines in the parallel-lines array. Experiment 2 tested whether the shift from assimilation to contrast of perceived length was determined by an absolute (foveal) measure of spatial separation or by relative separation of the contextual and test lines.

\section{EXPERIMENT 1}

The pool-and-store model predicts a shift from length assimilation to length contrast when contextual and test stimuli are sufficiently distant to be sampled sequentially. Additionally, the model is explicit in proposing that perceptual pooling, and hence assimilation, is produced when the intercontour separation among elements in an array is subfoveal. Conversely, suprafoveal spatial separations should produce storing, and hence contrast, due to the sequential sampling of the elements in the array. Precise definition of the size of the fovea is difficult (Ditchburn, 1973), but estimates range from $2^{\circ}$ (Ditchburn, 1973) to $5^{\circ}$ (Brown, 1975).

The general finding of assimilation with small spatial separations of contextual and test stimuli in the parallellines array is consistent with the foveal criterion. For example, Brigell and Uhlarik (1979) reported assimilation with a $5-\mathrm{mm}$ vertical spatial separation of horizontally oriented contextual and test lines. At their 40 -cm viewing distance, this intercontour separation was less than $1^{\circ}$. However, a more complete test of the pool-and-store model would include spatial separations that are clearly suprafoveal to determine whether a reversal to length contrast occurs. The present experiment included four levels of spatial separation of the contextual and test contours in the parallel-lines array; two of these separations were subfoveal and two were clearly suprafoveal. According to the pool-and-store model, assimilation should be observed with the smaller separations and contrast with the larger separations.

\section{Method}

Observers. Twenty members of an introductory psychology course at San Jose State University participated in the experiment in order to earn course credit. The observers were required to have at least $20 / 30$ visual acuity with or without correction. All observers were run in individual sessions.

Stimuli and Design. The stimulus lines were made with black tape oriented horizontally on a white background. The stimuli were then photographed and the resulting 35-mm slides were projected on the rear of a translucent screen by a Kodak random-access projector.

The specific contextual lengths for this experiment were chosen to produce maximum over- and underestimation of test length. Brigell and Uhlarik (1979) reported maximum distortion of test length when the ratio of contextual- to test-line length (the framing ratio) was around 1.67 , or its inverse. The projected lengths of the contextual lines used in the present experiment were 30, 40, 90 , and $120 \mathrm{~mm}$ and the test line was $60 \mathrm{~mm}$, resulting in framing ratios of $0.5,0.67,1.5$, and 2.0. The thickness of the lines on the viewing screen was $1.5 \mathrm{~mm}$ and the viewing distance was $40 \mathrm{~cm}$.

There were four levels of vertical spatial separation of the contextual and test lines on the slides: $5,25,75$, and $100 \mathrm{~mm}$. At the 40-cm viewing distance, the visual angles of spatial separation at these four levels were $0.7^{\circ}, 3.6^{\circ}, 10.7^{\circ}$, and $14.3^{\circ}$, respectively. Thus, two of the spatial separations resulted in arrays with subfoveal spatial separation, or close to it $\left(0.7^{\circ}\right.$ and $\left.3.6^{\circ}\right)$, and two resulted in arrays with suprafoveal separation $\left(10.7^{\circ}\right.$ and $\left.14.3^{\circ}\right)$. The $60-\mathrm{mm}$ test line was centered on the viewing screen and the contextual line was centered $5,25,75$, or $100 \mathrm{~mm}$ above and parallel to the test line. To summarize the design of the stimuli, there were four levels of contextual length $(30,40,90$, and $120 \mathrm{~mm}$ ) and four levels of spatial separation $(5,25,75$, and $100 \mathrm{~mm})$, resulting in 16 test slides.

Test-line judgments were obtained using a graded serjes comparison scale (cf. Coren \& Girgus, 1972). The scale was located $15 \mathrm{~cm}$ to the right of the midpoint of the test line. It consisted of 11 horizontally oriented lines, labeled $\mathrm{A}$ through $\mathrm{K}$, which ranged from 50 to $70 \mathrm{~mm}$ in $2-\mathrm{mm}$ increments. Therefore, the point of objective equality (POE) on this scale was $F$, or $60 \mathrm{~mm}$, for all test stimuli. The lines on the graded series scale were separated vertically by $1 \mathrm{~cm}$. In order to encourage observers to use a wide range of responses, eight filler stimuli with test lengths ranging from 52 to $68 \mathrm{~mm}$ were included in the design. Responses to these filler stimuli were not analyzed.

To familiarize observers with the use of the graded series scale, there were also six practice stimuli, which consisted of only a test line (no contextual line). The lengths of these stimuli were 52,56, $60,60,64$, and $68 \mathrm{~mm}$.

Procedure. The observers viewed the projected image of the stimuli in an otherwise darkened room. They were familiarized with the range and variety of the stimuli, as well as with the use of the graded series scale. Specifically, they were told that their task was 
to match the apparent length of the test line to one of the 11 lines on the graded series scale by calling out to the experimenter the letter of the matching line. In the event that a test line appeared to be intermediate in length between two lines on the graded series scale, the observers were instructed to call out the letters for both lines.

After the instructions, each observer proceeded through a random order of the six practice stimuli. These practice trials were followed by two random orders of the 16 test and 8 filler stimuli. A trial consisted of a $5-\mathrm{sec}$ presentation of the test or filler slide containing contextual and test lines, during which the test judgment was to be made. Trials were separated by 10 -sec dark intervals. In all, each of the 20 observers made 54 test judgments: 6 practice judgments and 2 judgments of each of the 16 test and 8 filler stimuli. The entire procedure required approximately $15 \mathrm{~min}$ for each observer.

\section{Results and Discussion}

The responses to the 60 -mm no-context test lines contained in the practice series were averaged to give an estimate of the point of subjective equality (PSE) of the 60$\mathrm{mm}$ test line contained in the test stimuli. The mean of the 40 responses to this control line (2 responses from each of 20 observers) was $56.7 \mathrm{~mm}$. Test-line judgments were converted into deviations from PSE by subtracting the PSE from the mean judged length of each test stimulus. These data are presented in Figure 2. Clearly, there was assimilation of test length when the contextual and test stimuli were spatially proximal (see curve labeled " $5 \mathrm{~mm}$ "). At this 5-mm $\left(0.7^{\circ}\right)$ spatial separation, con-

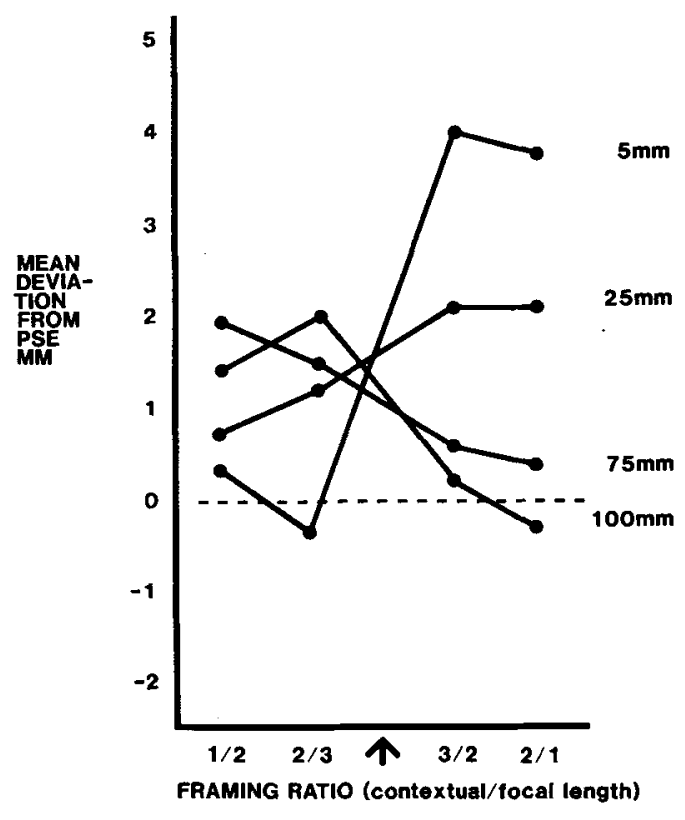

Figure 2. Mean deviations from the point of subjective equality (PSE), in millimeters, for the 5-, 25-, 75-, and 100-mm spatial separation conditions of Experiment 1. The 5-mm spatial separation data clearly indicate assimilation of perceived test length and the $100-\mathrm{mm}$ separation data indicate contrast. The abscissa indicates the ratio of contextual- to test-line length (the framing ratio) for the test stimuli. The arrow on the abscissa indicates the transition from framing ratios $<1$ to ratios $>1$. textual lines longer than the $60-\mathrm{mm}$ test line $(90$ and $120 \mathrm{~mm}$ ) produced overestimation of the test line. At the other extreme, length contrast resulted when the contextual and test stimuli were spatially distal (see curve labeled " $100 \mathrm{~mm}$ "). For this 100 -mm $\left(14.3^{\circ}\right)$ spatial separation, overestimation of perceived test length was produced by contextual lines shorter than the $60-\mathrm{mm}$ test line $(30$ and $40 \mathrm{~mm})$. For both effects, underestimation either was not present or was trivial. This is not surprising, since many studies of length distortion have found that overestimation of perceived length is much greater than underestimation (Brigell \& Uhlarik, 1979; Brigell, Uhlarik, \& Goldhorn, 1977; Jordan \& Uhlarik, 1985, 1986) and that underestimation of perceived test length is nonsignificant (Pollack \& Chaplin, 1964). Brigell et al. (1977) proposed that this asymmetry is produced by logarithmic representation of length in the visual system. The data for the other two curves (25 and $75 \mathrm{~mm}$ ) are less clear, but for the $25-\mathrm{mm}\left(3.6^{\circ}\right)$ spatial separation, distortions of perceived length were in the direction of assimilation, whereas the $75-\mathrm{mm}\left(10.7^{\circ}\right)$ separation produced distortions in the direction of length contrast.

The data for each level of spatial separation were analyzed separately in 4 (contextual lengths) $\times 2$ (replications) $\times 20$ (observers) within-subjects analyses of variance. In all four analyses, the effect of contextual length was statistically significant $[F(3,57) \geq 3.16, p<.05]$, indicating that contextual line length affected test judgments. Again, for the two smaller spatial separations, 5 and $25 \mathrm{~mm}\left(0.7^{\circ}\right.$ and $\left.3.6^{\circ}\right)$, the direction of the distortion indicates length assimilation, whereas the larger spatial separations, 75 and $100 \mathrm{~mm}\left(10.7^{\circ}\right.$ and $\left.14.3^{\circ}\right)$, seem to produce length contrast.

In summary, the present experiment demonstrated that under conditions of clearly suprafoveal vertical separation of horizontal contextual and test contours, length assimilation reverses to length contrast. This finding is supportive of the pool-and-store model of size distortion and points to the limitations of any model of length distortion that does not make provision for both assimilation and contrast.

\section{EXPERIMENT 2}

The results of Experiment 1 were generally supportive of Girgus and Coren's (1982) proposal that assimilation is produced under conditions of subfoveal separation of contextual and test stimuli in the parallel-lines array and that contrast results from suprafoveal spatial separation. This foveal criterion of spatial separation along the orthogonal dimension might serve as a useful means of classifying size distortions, on an a priori basis, as either assimilation or contrast effects.

At the same time, the results of Experiment 1 did not specifically rule out alternative models of size distortion. For example, the data were also consistent with the attentive-field postulate of Pressey's assimilation theory (Pressey \& Murray, 1976). This postulate states that as- 
similation magnitude decreases as a contextual stimulus moves from the center to the periphery of the observer's attentive field. Precise operational specification of the attentive field has proven difficult (see Bross, Blair, \& Longtin, 1978; Pressey, 1979), but Pressey and DiLollo (1978) have suggested that the attentive field's size changes as a constant ratio of target- or test-line size. If this suggestion is correct, and if assimilation occurs only within the attentive field, then the spatial separation required to result in a shift from assimilation to contrast might not depend as heavily on some absolute (foveal) criterion of spatial separation as Girgus and Coren (1982) suggest. Rather, the spatial separation needed to produce the shift might depend more on the relative separation of contextual and test elements in an array.

It is not possible to determine whether it was absolute or relative spatial separation that was responsible for the shift from length assimilation to contrast noted in Experiment 1 , because they covaried; as spatial separation varied from sub- to suprafoveal, relative separation also varied. The purpose of Experiment 2 was to remove this confound of interpretation of the results of Experiment 1 by holding relative spatial separation constant while varying absolute separation. This was accomplished by replicating Experiment 1 in all respects but one: the entire size of the test arrays was reduced so that all four levels of spatial separation were subfoveal (or close to it, given the imprecision of definition). This manipulation had the net effect of varying absolute spatial separation while leaving the relative spatial locations of contextual and test stimuli the same as they were in Experiment 1 . If the data failed to indicate a shift from assimilation to contrast under these conditions of subfoveal spatial separation, the foveal criterion of spatial separation of the pool-and-store model would be supported. If, however, the shift from assimilation to contrast observed in Experiment 1 occurred under these conditions of subfoveal spatial separation, it would point to the importance of the relative spatial separation of contextual and test stimuli.

\section{Method}

Observers. Twenty members of an introductory psychology course at San Jose State University participated in the experiment in order to earn course credit. The observers were required to have at least $20 / 30$ visual acuity with or without correction. All observers were again run in individual sessions.

Stimuli and Design. The stimuli and design of this experiment were identical to those of Experiment 1, with the following exceptions. The entire array was reduced by $70 \%$, resulting in test lengths of $18 \mathrm{~mm}$ and contextual lengths of $9,12,27$, and $36 \mathrm{~mm}$. Thus, the framing ratios were the same as in Experiment $1(0.5,0.67$, 1.5 , and 2.0). Under these conditions the four levels of spatial separation were reduced to $1.5,7.5,22.5$, and $30 \mathrm{~mm}$. Due to the resolution limits of the lens on the slide projector, the viewing distance was increased to $500 \mathrm{~mm}$ to produce the following four visual angles of spatial separation: $0.17^{\circ}, 0.85^{\circ}, 2.55^{\circ}$, and $3.4^{\circ}$. The thickness of the lines on the screen was reduced to $0.4 \mathrm{~mm}$. The graded series comparison scale was now located $4.5 \mathrm{~cm}$ to the right of the midpoint of the test line, and the lines on the scale ranged from $15 \mathrm{~mm}$ to $21 \mathrm{~mm}$ in $0.6-\mathrm{mm}$ increments. The POE was still $\mathrm{F}$, or
$18 \mathrm{~mm}$, and the lines were separated vertically by $3 \mathrm{~mm}$. Finally, the lengths of the practice stimuli were $15.6,16.8,18.0,18.0,19.2$, and $20.4 \mathrm{~mm}$.

Procedure. The procedure was identical in all respects to that of Experiment 1.

\section{Results and Discussion}

The responses to the 18-mm no-context test lines contained in the practice series were averaged to give an estimate of the PSE of the 18-mm test line contained in the test stimuli. The mean of the 40 responses to this control line ( 2 responses from each of 20 observers) was $16.9 \mathrm{~mm}$. Test-line judgments were converted into deviations from PSE by subtracting the PSE from the mean judged length of each test stimulus. These data are presented in Figure 3. Despite differences in the absolute amount of distortion, these data are clearly similar to those of Experiment 1. Thus, there was assimilation of perceived test length at the smallest spatial separation $\left(1.5 \mathrm{~mm}\right.$ or $0.17^{\circ}$; see curve labeled " $1.5 \mathrm{~mm}$ "). At the other extreme, length contrast was produced in the largest spatial separation condition ( $30 \mathrm{~mm}$ or $3.4^{\circ}$; see curve labeled "30 mm"). Also as in Experiment 1, the data for the intermediate spatial separations were less clear, but for the $7.5-\mathrm{mm}\left(0.85^{\circ}\right)$ separation, the length distortions were in the direction of assimilation, whereas the 22.5 $\mathrm{mm}\left(2.55^{\circ}\right)$ separation condition produced distortions in the direction of length contrast.

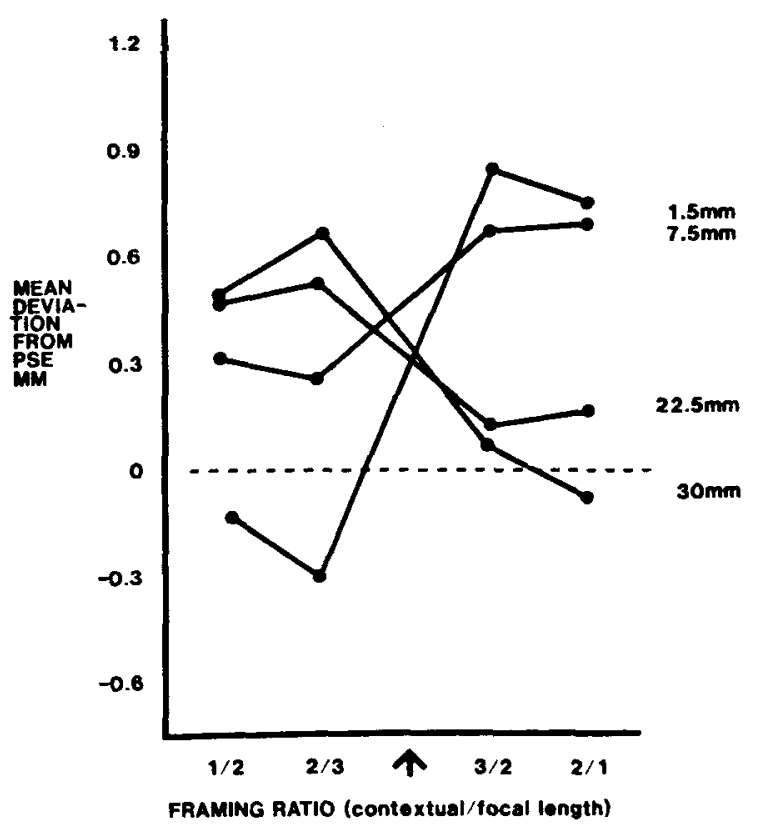

Figure 3. Mean deviations from the point of subjective equality (PSE), in millimeters, for the 1.5-, 7.5-, 22.5-, and 30-mm spatial separation conditions of Experiment 2. The 1.5-mm spatial separation data clearly indicate assimilation of perceived test length and the 30-mm separation data indicate contrast. The abscissa indicates the ratio of contextual- to test-line length (the framing ratio) for the test stimuli. The arrow on the abscissa indicates the transition from framing ratios $<1$ to ratios $>1$. 
The data for each level of spatial separation were analyzed separately in 4 (contextual lengths) $\times 2$ (replications) $\times 20$ (observers) within-subjects analyses of variance. The effect of contextual length was statistically significant for the $1.5-\mathrm{mm}\left(0.17^{\circ}\right), 22.5-\mathrm{mm}\left(2.55^{\circ}\right)$, and $30-\mathrm{mm}\left(3.4^{\circ}\right)$ spatial separation conditions $[F(3,57)$ $\geq 4.40, p<.01]$, indicating that contextual line length affected test judgments. For the $1.5-\mathrm{mm}\left(0.17^{\circ}\right)$ condition, the direction of the effect was clearly indicative of assimilation, whereas for the $30-\mathrm{mm}\left(3.4^{\circ}\right)$ condition the effect was one of contrast. The main effect of contextual length did not reach statistical significance in the $7.5-\mathrm{mm}$ $\left(0.85^{\circ}\right)$ spatial separation condition.

The present experiment demonstrates the importance of the relative spatial separation of contextual and test contours in determining whether length assimilation or length contrast occurs. This indicates that any criterion based on the size of the fovea is not useful for predicting assimilation or contrast effects, nor is any other criterion based on absolute spatial separation. As an example, one level of spatial separation of contextual and test stimuli used in Experiment 1 was $25 \mathrm{~mm}\left(3.6^{\circ}\right)$, and assimilation resulted. On the other hand, the $30-\mathrm{mm}$ or $3.4^{\circ}$ of spatial separation used in Experiment 2 resulted in contrast. Thus, the distortion was reversed, although the absolute spatial separations were very similar. What did change, however, was the relative separation of contextual and test stimuli. It seems that $3.4^{\circ}$ of separation from an $18-\mathrm{mm}$ test line is perceptually much greater than $3.6^{\circ}$ of separation from a $60-\mathrm{mm}$ test line. This is exactly what would be expected if attentive field size changes relative to test line size, as Pressey and DiLollo (1978) suggested. Thus, it may be that perceptual pooling occurs when contextual and test stimuli are relatively proximal, whereas storing results from relatively large spatial separations.

\section{GENERAL DISCUSSION}

The present study resulted in two important findings. First, simultaneous length contrast was produced in an array that had previously produced only simultaneous assimilation. Second, we determined that the reversal from simultaneous assimilation to simultaneous contrast was contingent on relative, rather than absolute, spatial separation of contextual and test stimuli in the parallel-lines array. Each of these findings will be discussed in turn.

\section{Simultaneous Length Contrast}

The pool-and-store model proposes that any experimental manipulation that encourages an observer to sequentially sample the elements in an array will result in storing, and hence in contrast. This implies a functional equivalence of temporal and spatial separation in producing contrast. In the temporal domain, Jordan and Uhlarik (1985) have demonstrated that either 5 or $60 \mathrm{sec}$ of exposure to the contextual line prior to a test judgment produces length contrast in the parallel-lines array. Additionally, Jordan and Uhlarik (1986) demonstrated length contrast in the Müller-Lyer figure when the (contextual) fins were inspected before the (test) shaft was judged. In our present attempt to demonstrate simultaneous length contrast produced by spatial separation of contextual and test lines, we had to address two issues: the dimension along which to spatially separate the stimuli and the amount of spatial separation.

Brigell and Uhlarik (1979) and Schiano and Girgus (1981) reported that extremely large or extremely small contextual lines were not effective in producing length contrast; with very large or very small contextual lines, assimilation was reduced to no distortion. This indicates that the spatial separation from the tip of the test line to the tip of the contextual line is not the dimension responsible for producing a shift from pooling to storing. For example, at the $7: 1$ framing ratio $(420 \mathrm{~mm}$ contextual length $/ 60 \mathrm{~mm}$ test-line length) used by Schiano and Girgus, the horizontal distance from the left tip of the test line to the left tip of the contextual line was $180 \mathrm{~mm}$, or over $25^{\circ}$ of visual angle at their $40-\mathrm{cm}$ viewing distance. Thus, extreme spatial separations of contextual and test lines along the dimension parallel to the test line did not promote sequential sampling of the array. This finding also indicates that the absolute size of the stimulus elements in the array is not critical in determining the shift from assimilation to contrast, because the extremely large stimuli produced assimilation. If the region defined by the fovea does represent the area within which pooling, and hence assimilation, occurs, then the absolute size of the array cannot be the criterion for determining the shift from assimilation to contrast. In Schiano and Girgus's study, many fixations would have been required to sample all of the $420-\mathrm{mm}$ (or about $60^{\circ}$ at the $40-\mathrm{cm}$ viewing distance) contextual line; regardless of where they initially fixated, observers would have had to move their eyes in order to sample the entire line. Given these multiple fixations, length contrast would have been expected, rather than the observed assimilation. An alternative criterion suggested by Girgus and Coren (1982) is that intercontour separation along the dimension orthogonal to the test line promotes sequential sampling. Support for this alternative was provided by Jordan and Uhlarik (1986), who demonstrated simultaneous length contrast produced by vertical separation of the (contextual) fins from the (test) shaft in a horizontally oriented Müller-Lyer figure.

The second issue was the amount of spatial separation needed to produce contrast. Girgus and Coren (1982) proposed that a shift from assimilation to contrast is under the control of absolute intercontour separation. Subfoveal intercontour separations promote pooling, and hence assimilation, and suprafoveal separations promote storing, and hence contrast. Thus, as long as the intercontour separations are subfoveal, parts of both the contextual and test lines will be sampled whenever the test line is fixated. According to the model, this stimulus situation produces assimilation; it is not necessary for the entire stimulus array to be imaged within the fovea.

On the basis of the above considerations, we attempted in Experiment 1 to demonstrate simultaneous length contrast in the parallel-lines array by employing a suprafo- 
veal vertical spatial separation of horizontal contextual and test lines. Our observation of length contrast under these conditions is supportive of a pool-and-store approach to size distortions and points to the limitations of any model that describes the parallel-lines array solely in terms of assimilation.

\section{Relative Spatial Separation}

Although Experiment 1 provided support for a unified pool-and-store model, it did not specifically rule out any other models of perceptual distortion. For example, the manipulation of absolute spatial separation necessarily resulted in a manipulation of relative separation. This is a critical problem, because assimilation theory (e.g., Pressey \& DiLollo, 1978) proposes that assimilation occurs only within an observer's attentive field and that attentive field size changes as a constant ratio of test length. It is implicit in the theory that stimuli outside the attentive field are not part of the context, and thus cannot be glimpsed simultaneously (see Pressey et al., 1971). The important point is that the alternative possibility of relative spatial separation's determining the shift from assimilation to contrast could not be ruled out by Experiment 1 . Experiment 2 provided a stronger test of the pool-andstore model by replicating Experiment 1, but with modifications, so that all spatial relations among stimuli were identical to those in Experiment 1 but the absolute spatial separations were all subfoveal. The occurrence of simultaneous length contrast under these conditions of subfoveal separation indicates that relative spatial separation is more important than absolute spatial separation, and that pooling and storing are determined more by the relations among stimuli in the attentive field of the observer than by the fovea.

\section{A Revised Pool-and-Store Model}

The results of the present study suggest a model that is capable of specifying an a priori classification system of visual size distortion. The model is essentially the pooland-store model of Girgus and Coren (1982), with one major revision, as suggested by Pressey's assimilation theory (Pressey \& DiLollo, 1978; Pressey \& Wilson, 1980). This revised model proposes that the visual information processing system imposes constraints on the number of elements in an array that can be apprehended simultaneously by an observer. Elements in an array that are spatially proximal tend to be perceptually pooled, and the result is often assimilation; spatially or temporally distal elements are sampled sequentially, leading to encoding of differences, with contrast the result. The criteria for determining whether elements in an array are spatially proximal or spatially distal are the dimension along which stimuli are spatially separated and the size of the test stimulus. Spatial separation along the dimension orthogonal to the test stimulus (in this case, vertical separation of horizontally oriented stimuli) results in a shift from assimilation to contrast (see Girgus \& Coren, 1982; Pressey \& Wilson, 1980). Importantly, the amount of spa- tial separation needed to produce this shift is related to the size of the test stimulus; smaller test stimuli require a smaller spatial separation to produce sequential processing of contextual and test stimuli than do larger test stimuli (Pressey \& DiLollo, 1978).

Analysis of simultaneous length contrast effects in the Müller-Lyer figure (Jordan \& Uhlarik, 1986) and the parallel-lines figure (Experiments 1 and 2) reveals that whenever the spatial separation of contextual and test stimuli was greater than the test length, contrast was observed. For example, a $100-\mathrm{mm}$ spatial separation of a contextual line from a $60-\mathrm{mm}$ test line resulted in contrast (Experiment 1), as did a 30-mm separation of a contextual line from an 18-mm test line (Experiment 2). On the other hand, when the spatial separation was less than the test length, assimilation was observed; a $25-\mathrm{mm}$ spatial separation of a contextual line from a $60-\mathrm{mm}$ test line produced assimilation (Experiment 1). The important point is that the occurrence of assimilation or contrast depended on both spatial separation and test-line length, rather than the absolute criterion of spatial separation predicted by the pool-and-store model.

The revised model proposes that assimilation occurs when the centers of the contextual and test stimuli are within an attentive field of roughly circular shape (Pressey \& DiLollo, 1978), and that the radius of the attentive field is the length of the test line. Conversely, contrast results when the center of the contextual line falls outside the attentive field defined by the test line (Girgus \& Coren, 1982; Pressey \& Wilson, 1980). Although admittedly post hoc, this hypothesis is precise enough to be tested in a parametric study of spatial separation.

\section{REFERENCES}

Brigell, M., UHLARIK, J. (1979). The relational determination of length illusions and length aftereffects. Perception, 8, 187-197.

Brigell, M., UhlariK, J., Goldhorn, P. (1977). Contextual influences on judgments of linear extent. Journal of Experimental Psychology: Human Perception \& Performance, 3, 105-118.

Bross, M., Blatr, R., \& Longtin, P. (1978). Assimilation theory, attentive fields and the Mueller-Lyer illusion. Perception, 7, 297-304.

Brown, T. S. (1975). General biology of sensory systems. In B. Scharf (Ed.), Experimental sensory psychology (pp. 68-111). Glenview, IL: Scott, Foresman.

Coren, S., Girgus, J. S. (1972). A comparison of five methods of illusion measurement. Behavior Research Methods \& Instrumentation, 4, 240-244.

Coren, S., Graus, J. S. (1978). Seeing is deceiving: The psychology of visual illusions. Hillsdale, NJ: Erlbaum.

DITCHBURN, R. W. (1973). Eye-movements and visual perception. OXford: Clarendon Press.

GIRGUS, J. S., CoREN, S. (1982). Assimilation and contrast illusions: Differences in plasticity. Perception \& Psychophysics, 32, 555-561.

JoRdan, K., \& Uhlarik, J. (1985). Assimilation and contrast of perceived length depend on temporal factors. Perception \& Psychophysics, $37,447-454$.

Jordan, K. , Uhlarik, J. (1986). Length contrast in the Müller-Lyer figure: Functional equivalence of temporal and spatial separation. Perception \& Psychophysics, 39, 267-274.

Pollack, R. H., \& Chaplin, M. R. (1964). Effects of prolonged stimulation by components of the Mueller-Lyer figure upon the magnitude of the illusion. Perceptual \& Motor Skills, 18, 377-382. 
Pressey, A. W. (1972). The assimilation theory of geometric illusions: An additional postulate. Perception \& Psychophysics, 11, 28-30.

Pressey, A. W. (1979). Are black circles attentive fields? A reply to Bross, Blair, and Longtin. Perception, 8, 237-238.

Pressey, A. W. (1983). Some delusions about illusions. Invited address at Kansas State University, Manhattan, KS.

Pressey, A. W., Bross, M. (1973). Assimilaton theory and the reversed Mueller-Lyer illusion. Perception, 2, 211-217.

Pressey, A. W., Butchard, N., \& SCrIvner, L. (1971). Assimilation theory and the Ponzo illusion: Quantitative predictions. Canadian Journal of Psychology, 25, 486-497.
Pressey, A. W., \& Dilollo, V. (1978). Effects of distance between standard and comparison lines on the Müller-Lyer illusion. Perception \& Psychophysics, 24, 415-419.

Pressey, A. W., \& Murray, R. (1976). Further developments in the assimilation theory of geometric illusions: The adjacency principle. Perception \& Psychophysics, 19, 536-544.

Pressey, A. W., \& Wilson, A. E. (1980). Assimilation theory and the Baldwin illusion. Italian Journal of Psychology, 7, 65-73.

SCHIANo, D. J., \&IRGUS, J. S. (1981). A second look at the parallel line illusion. Paper presented at the meeting of the Eastern Psychological Association, New York.

(Manuscript received May 23, 1986;

revision accepted for publication September 15, 1986.) 